\title{
Effect of Protocolized Weaning and Spontaneous Breathing Trial vs Conventional Weaning on Duration of Mechanical Ventilation: A Randomized Controlled Trial
}

\author{
Rashmi Kishore $^{1} \odot$, Urmila Jhamb ${ }^{2}$
}

\begin{abstract}
Background: Identifying ventilated patients ready for extubation is a challenge for clinicians. Premature extubation increases risks of reintubation while delayed weaning increases complications of prolonged ventilation. We compared the duration of mechanical ventilation (MV) and extubation failure in children extubated using a weaning protocol based on pressure support spontaneous breathing trial (PS SBT) vs those extubated after nonprotocolized physician-directed weaning.

Patients and methods: A prospective randomized controlled trial was conducted in the pediatric intensive care unit of a tertiary care hospital in children ventilated for $\geq 24$ hours. All eligible patients underwent daily screening and were randomized once found fit. The intervention group underwent PS SBT of 2 hours duration followed by a T-piece trial and extubation. Controls underwent conventional weaning with synchronized intermittent mandatory ventilation mode and a T-piece trial before extubation.

Results: Eighty patients were randomized into two groups of 40 each. About $77.5 \%$ of patients passed the PS SBT on the first attempt. No statistical difference was found either in the duration of MV between the two groups [median (interquartile range) in days: 4.77 (2.89, 9.46$)$ in controls and $4.94(2.23,6.35)$ in cases, $p=0.62$ ] or in the rate of extubation failure $(13 \%$ and $10.5 \%, p=1)$. Mortality was found to be significantly higher in the reintubated patients compared to those not reintubated in both groups ( $p=0.002$ in cases and 0.005 in controls).

Conclusion: Weaning using PS SBT-based protocol though did not shorten the duration of MV, it was found to be safe for assessing extubation readiness and did not increase extubation failure (CTRI no-CTRI/2018/04/013270).

Keywords: Extubation failure, Extubation readiness trial, Mechanical ventilation, Spontaneous breathing trial, Weaning.

Indian Journal of Critical Care Medicine (2021): 10.5005/jp-journals-10071-23944
\end{abstract}

\section{HighLights}

- Protocolized weaning using daily screening and pressure support spontaneous breathing trial (PS SBT) did not shorten the duration of ventilation.

- The majority of the patients $(77.5 \%)$ passed the PS SBT on the first attempt and could be safely extubated without increasing extubation failure.

\section{INTRODUCTION}

Mechanical ventilation (MV) is an important lifesaving intervention frequently used in critically ill children but identifying ventilated patients ready for extubation still remains a challenge for clinicians. Premature extubation increases risks of reintubation, associated with a poorer prognosis, while delayed weaning and extended period of MV increases complications of prolonged ventilation. ${ }^{1-3}$

A significant proportion of patients being evaluated for weaning are ready for extubation. ${ }^{4-6}$ The process of weaning or reduction in the ventilatory support could be empirical, based on clinical judgment, or protocolized using spontaneous breathing trial (SBT). SBT, also referred to as extubation readiness trial (ERT), is performed on a patient while still intubated and evaluates the patient's cardiorespiratory tolerance to maintain spontaneous breathing with minimal or no support. 6,7 Pressure support (PS), continuous positive airway pressure (CPAP), and T-piece are three of the commonly used SBTs. ${ }^{7,8}$ Studies in adults

\begin{abstract}
${ }^{1,2}$ Department of Pediatrics, Maulana Azad Medical College and Lok Nayak Hospital, Delhi, India

Corresponding Author: Rashmi Kishore, Department of Pediatrics, Maulana Azad Medical College and Lok Nayak Hospital, Delhi, India, Phone: +91 7065197192/+91 7543042110, e-mail: rashmi.k186@ gmail.com

How to cite this article: Kishore R, Jhamb U. Effect of Protocolized Weaning and Spontaneous Breathing Trial vs Conventional Weaning on Duration of Mechanical Ventilation: A Randomized Controlled Trial. Indian J Crit Care Med 2021;25(9):1059-1065.
\end{abstract}

Source of support: Nil

Conflict of interest: None

have shown that weaning protocol with daily evaluation and use of SBT leads to a reduction in MV duration, treatment costs, and treatment-associated complications. ${ }^{6,9-11}$ However a few studies done in pediatric population have shown variable results, and their applicability in resource-limited countries still needs evaluation. ${ }^{12,13}$

In our study, we evaluated if protocolized weaning using daily screening of patients and use of pressure support spontaneous breathing trial (PS SBT) for assessing extubation readiness could lead to any reduction in ventilation duration compared to conventional weaning without increasing risks of premature extubation. 


\section{Materials and Methods}

Design: A single-center, open-labeled randomized controlled trial. Setting: Pediatric intensive care unit (PICU) of a tertiary care hospital in Delhi, India.

Study period: From April 2015 to March 2016.

Participants and sample size: Eligible patients were children, 1 month to 12 years of age, ventilated for $>24$ hours in PICU. Exclusion criteria were neuromuscular disorders, spinal cord injury above lumbar region, upper airway obstruction, tracheostomy, anatomical obstruction of lower airways, diaphragmatic hernia or palsy, congenital cyanotic heart disease, chronically ventilated patients ( $>3$ weeks), primary pulmonary hypertension, and significantly decreased lung capacity. A sample size of 80 patients with 40 in each group was taken for the study.

Recruitment and randomization: Patients were randomized into two groups by block randomization with each block of eight, using a computer-generated randomization list.

- Group A (control group): Received conventional physiciandirected weaning (No PS SBT).

- Group B (intervention group): Intervention, PS SBT, was given on fulfillment of eligibility criteria.

Allocation concealment: Sequentially arranged sealed opaque envelopes, prepared by a person who was not involved in the study, were used for allocation concealment.

Outcomes: Primary outcome-duration of MV. Secondary outcome-extubation failure (reintubation or unplanned noninvasive ventilation within 48 hours of extubation).

Methodology: All patients meeting the eligibility criteria were assessed daily for SBT eligibility. Once fit, they were enrolled in the study and randomized. Eligibility criteria for performing SBT were (i) hemodynamically stable patients with no or minimal inotropic support (dopamine/dobutamine $\leq 10 \mu \mathrm{g} / \mathrm{kg} / \mathrm{minute}$ ), (ii) basic disease pathology significantly improved/ resolved, (iii) presence of spontaneous breathing efforts, (iv) presence of gag or cough reflex on suctioning, (v) acceptable level of consciousness, (vi) $\mathrm{pH}$ 7.32 to 7.47 on recent blood-gas analysis done in last 12 hours, (vii) positive end expiratory pressure $\leq 5 \mathrm{~cm} \mathrm{H}_{2} \mathrm{O}$, fraction of oxygen in inspired air $\left(\mathrm{FiO}_{2}\right) \leq 0.5$, peak inspiratory pressure (PIP) $\leq 25$, (viii) absence of new infiltrates on the chest radiograph, (ix) no clinical need to increase ventilator support in the last 12 hours, $(x)$ no planned operative procedures requiring heavy sedation in the next 48 hours, and (xi) not on paralysis in the past 24 hours and minimal sedation ( $\leq$ midazolam $1 \mu \mathrm{g} / \mathrm{kg} /$ minute). These criteria provided a check sheet to see if factors contributing to ventilator dependence were reversed and to ensure objective and subjective measurement of patient's clinical stability, oxygenation, pulmonary function, and mental status, to assess suitability for weaning and extubation. ${ }^{12-14}$

In the control group, weaning was done as per the treating team's discretion without any written protocol. Most of our patients were ventilated using pressure control synchronized intermittent mandatory ventilation (SIMV) mode with pressure support. In the control arm, first the $\mathrm{FiO}_{2}$ was reduced up to 35 to $45 \%$, and PIP was reduced to $<20$, while maintaining oxygen saturation (93-99\%). Respiratory frequency was reduced slowly up to 8 to 10 in infants and 5 to 6 in older children, and finally reducing and stopping PS while maintaining $\mathrm{O}_{2}$ saturation, $\mathrm{MV}$, total respiratory rate (RR) and end tidal $\mathrm{CO}_{2}\left(\mathrm{ETCO}_{2}\right)$. Prior to extubation, all patients were given a T-piece trial of 2 hours duration which was the standard of care in our PICU.

Sedation with midazolam and fentanyl was commonly used in our ICU for the initial few days followed by midazolam alone. The usual practice was to give sedation holiday daily in the morning. If the patient was on any midazolam infusion, it was stopped before attempting T-piece trial and extubation.

In the intervention group, PS SBT was given by changing the ventilator mode to pressure support ventilation (PSV) with PS adjusted for increasing ET size-3 to $3.5: 10 \mathrm{~cm} \mathrm{H}_{2} \mathrm{O}, 4$ to 4.5: $8 \mathrm{~cm} \mathrm{H}_{2} \mathrm{O}$ and $\geq 5: 6 \mathrm{~cm} \mathrm{H}_{2} \mathrm{O}$, similar to study by PALISI network. ${ }^{12}$ If the patient was able to maintain a tidal volume $>5 \mathrm{~mL} / \mathrm{kg}$ for 10 minutes, trial was continued for the next 2 hours or else the patient was removed from the test and placed back on previous ventilator settings.

The patient was classified as failing the test if anytime during the 2-hour period any of the following criteria was met-(i) RR outside the normal for the given age-group ( $<1$ year: $30-60 /$ minute, 1-3 years: $24-40 /$ minute, $4-5$ years: $22-34 /$ minute, $6-12$ years: $18-30 /$ minute), (ii) increase in heart rate (HR) $>20 \%$ over baseline, (iii) increase in work of breathing/accessory muscle use, (iv) tidal volume $\leq 5 \mathrm{~mL} / \mathrm{kg}$, (v) blood pressure falls to $<5$ th centile for age, (vi) saturation decrease $\leq 92 \%$, and (vii) $\mathrm{ETCO}_{2}$ rises $>10 \mathrm{~mm} \mathrm{Hg} /$ hour.

Patients passing the SBT were subjected to a second T-piece trial as we were not confident about the predictability of PS SBT for safe extubation. The patient was disconnected from the ventilator and allowed to breathe through a T-piece connected to ET tube (with added oxygen if required) and was monitored for the same criteria for 2 hours. If the patient passed both the tests, they were extubated. On failure of either of the test, the patient was put back on the ventilator with previous settings and reassessed again for SBT every day.

Extubated patients were monitored for any complications or need for reintubation. Reintubation was done in cases of extubation failure indicated by (i) marked increase in RR with severe retractions/ desaturation $<90 \%$ /apnea $>20$ seconds, (ii) blood-gas criteria $\left(\mathrm{PaO}_{2} / \mathrm{FiO}_{2}\right.$ ratio $<200$ or $\mathrm{PaCO}_{2}>50 \mathrm{~mm} \mathrm{Hg}$ with $\left.\mathrm{pH}<7.3\right)$, and (iii) inadequate respiratory effort with decreased consciousness or cardiovascular insufficiency.

Patients were enrolled only once during their hospital stay and only the first intubation was considered for analysis. Both groups were managed by the same medical team and weaning prior to randomization was similar in the groups. Daily screening of patients, randomization, and SBT in the intervention group was performed by a designated resident involved in the study, while extubation/reintubation and further management were done by the managing team.

Data collection: For all patients, the following variables were collected-age, sex, date of admission, primary diagnosis, comorbidities, indication for MV, date of intubation and start of MV, ET size, total duration of MV, ventilatory parameters, weaning time, SBT outcome (failure or success), T-piece outcome, patient outcome (extubation/death), and extubation failure, if therewith reason.

Data analysis: Data entry was done using a Microsoft Excel spreadsheet and analyzed using descriptive statistics/SPSS version 22. Results were expressed as proportions for categorical data and for continuous data as mean \pm SD for normally distributed variables or median [interquartile range (IQR)] for non-normally distributed variables. For categorical data, chi-square tests or Fisher's exact tests were used if the expected values in any single cell were $<5$. For continuous variables, Mann-Whitney $U$ test (for non-normal 
distribution) or Student's $t$-test (for normal distribution) was used. $p$-value $<0.05$ was considered significant.

Ethical considerations: The study was approved by the Institutional Ethics Committee. The details of the study were communicated to parents verbally and by a patient information sheet. Written informed consent was taken from parents/guardians of children before inclusion into the study. Parents were able to withdraw their child from the study at any time.

\section{Results}

A total of 80 patients were enrolled in the study with 40 in each group (Flowchart 1). The two groups were comparable in their characteristics except for median age and weight, which was significantly lower in the intervention group, and subsequently also differed in the ET tube sizes (Table 1).

A total of 37 out of 40 patients (92.5\%) in the intervention group passed PS SBT on one or more attempts. Thirty-one cases (77.5\%) passed the PS SBT in the first attempt, out of which 30 passed the consecutive T-piece trial and 29 of them got extubated (Supplementary file available on IJCCM journal website). One patient who was a case of postdiphtheritic palatal palsy with pneumonia could not be extubated despite passing both the trials as there was pooling of secretions and this patient had to be tracheostomized. One patient who only failed the T-piece trial passed both PS and T-piece trial the next day and was extubated.

Of the remaining nine patients who failed the first trial, one expired before the trial could be repeated. Six successfully passed both the trials on successive attempts and were extubated (four after second and one each after third and fifth attempt). Two cases failed PS SBT repeatedly. One was a case of meningitis with intracranial infarct, failed four PS trials, but extubation attempted as per physician's decision was successful. Second was a case of empyema thoracis, who passed the fourth and fifth PS SBT but failed the subsequent T-piece trial, got self-extubated and was successfully managed on oxygen support. A total of 58 PS SBT was given to 39 patients, out of which $39(67 \%)$ were successful and 19 (33\%) failed. The median number of days for which the patient had been ventilated before giving PS SBT was 2.71 (1.56-5.35) in those who passed the trial in the first attempt and 4.29 (2.58-5.67) in those who did not $(p=0.39)$. The mean duration after which the PS SBT trial failed was 23 minutes while the maximum was 1 hour, with tachypnea being the most common cause of failure.

In the control group, a case of postdiphtheritic polyneuropathy had difficult weaning attributed to neuromuscular weakness and required elective tracheostomy. Rest of the 39 patients were conventionally weaned and given T-piece trial which was passed by 36 patients on the first attempt (92.3\%), by two on the second attempt, and one failed the trial and expired before becoming eligible for a repeat trial. The median number of days for which the patient had been ventilated before taking on T piece was 3.79 $(1.90-8.27)$ in those who passed the trial and $1.29(1.18-2.14)$ in those who did not, which was not significantly different $(p=0.34)$. The clinical profile of patients failing various extubation trials is given in Table 2.

There was no difference in total extubation rates in the two groups, with 38 getting extubated in both. The mean duration of ventilation was similar in both groups (Table 3, Figure 2). They also did not differ with respect to weaning time (time from the beginning of weaning to extubation), duration of PICU stay, and duration of hospital stay. However total reintubation rate was lower in the intervention group as compared to controls but was not statistically significant [15.78\% (6/38) vs $26.3 \%(10 / 38), p=0.4]$. The rate of extubation failure, as defined by patients reintubated within 48 hours was $10.5 \%$ (4/38) and 13\% (5/38) in the intervention and control groups, respectively, and was not significantly different. Reintubation rate was lower, i.e., 13.8\% (4/29) in those extubated after passing the PS SBT on the first attempt as compared to $40 \%$ $(2 / 5)$ in those who were extubated after the second attempt. There were however no reintubations in those four who passed the trial

Flowchart 1: Flowchart depicting the outcome of PS SBT in the intervention group

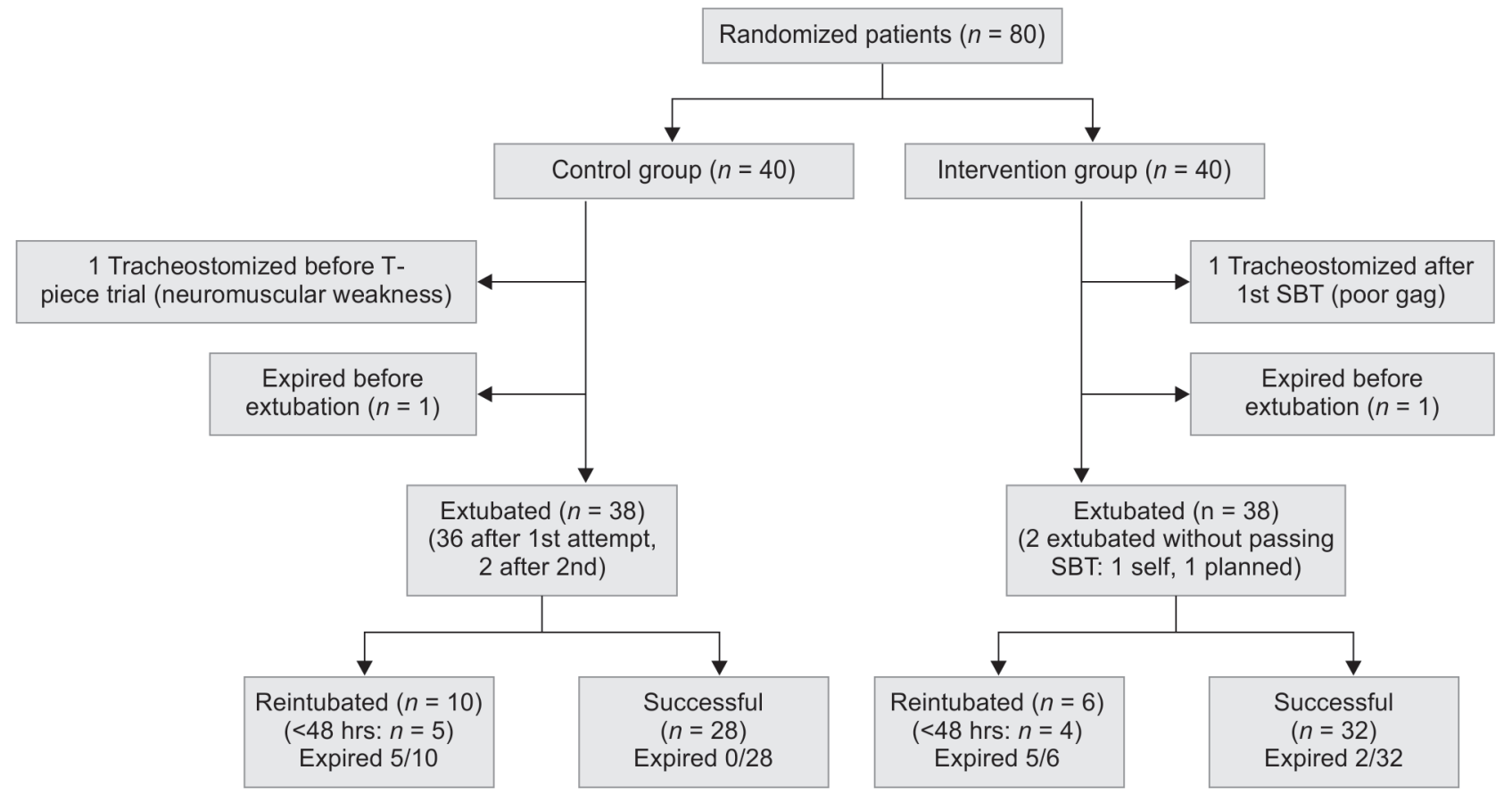



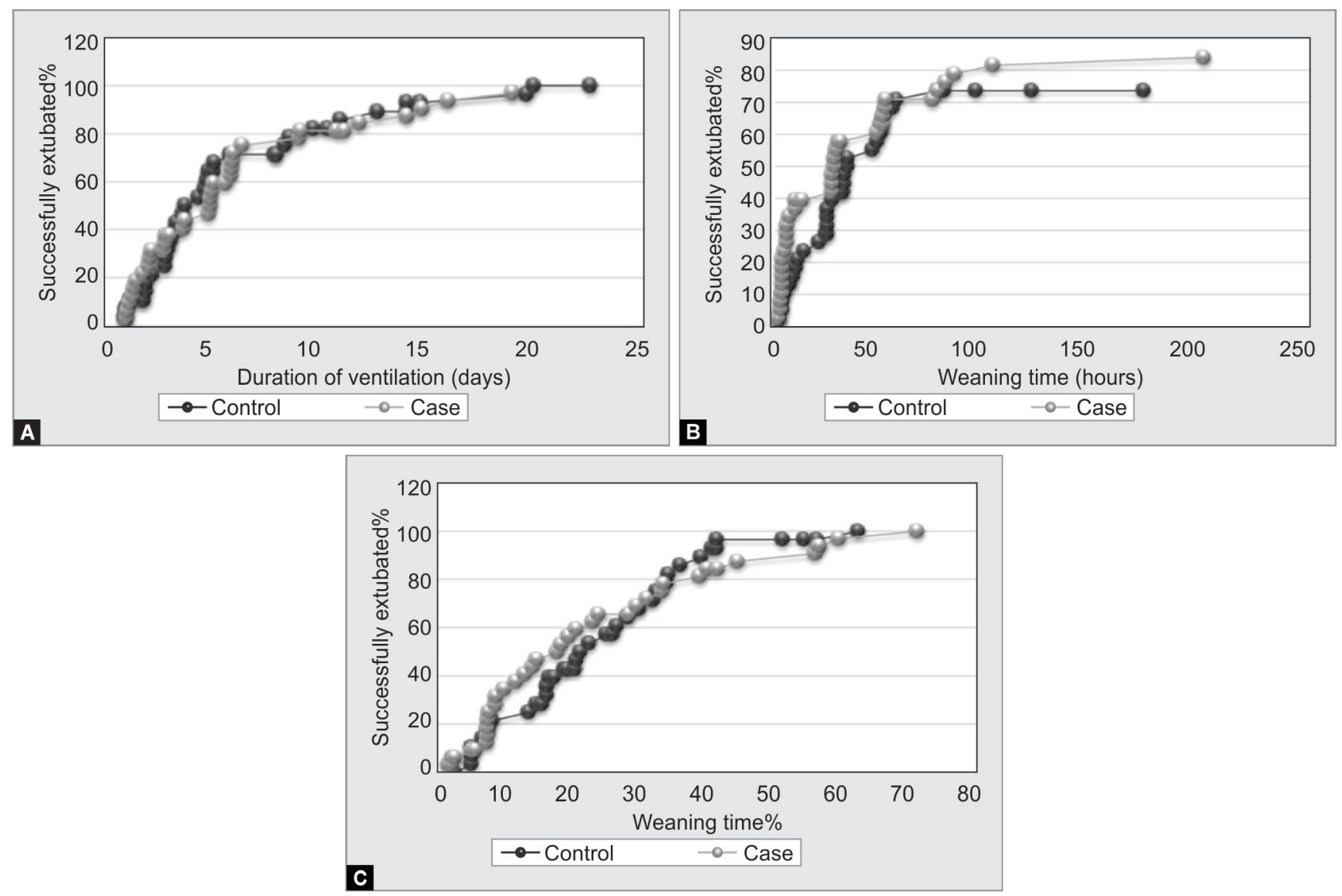

Figs $2 \mathrm{~A}$ to C: Kaplan-Meier curve comparing (A) duration of mechanical ventilation; (B) weaning time; and (C) weaning time as percentage of total MV duration between the two groups

later with more attempts. The number of patients in subsequent attempts of SBTs was so small that it is difficult to conclude whether the need for more attempts of SBTs is associated with more extubation failures.

The mean time to reintubation in extubation failures was 5.6 hours for controls and $\mathbf{1 7 . 5}$ hours for the intervention group, with the earliest being 1 hour in both groups. Of the reintubated patients, four in the control group needed tracheostomy, three for upper airway obstruction (all ventilated for $>10$ days), and one due to vocal cord palsy (an operated case of brain stem glioma). In the intervention group, two reintubated patients were tracheostomized for prolonged ventilation with difficult weaning. Noninvasive ventilation was not used after extubation in any patient.

There was no statistically significant difference in the duration of ventilation prior to extubation in reintubated patients compared to those who were not, in both groups. However, there was a significant difference in mortality between the patients successfully extubated vs those requiring reintubations (intervention group: $2 / 32$ expired in successfully extubated vs $5 / 6$ in reintubated, $p=0.0002$, controls: $0 / 28$ in successfully extubated vs $5 / 10$ in reintubated patients, $p=0.0005$ ).

\section{Discussion}

There is no clear consensus on the ideal PS to be used for PS SBT. Adult studies have used a uniform pressure of $7 \mathrm{~cm} \mathrm{H}_{2} \mathrm{O}$ while pediatric studies have used variable support. ${ }^{12-19}$ Studies have shown that PS of 3 to $4 \mathrm{~cm}$ may be sufficient to alleviate any increased resistance due to ET tube at physiological flow rates. ${ }^{20-22}$ Khemani et al. found very low work of breathing in children while on PS of $10 \mathrm{~cm} \mathrm{H}_{2} \mathrm{O}$ measured by an esophageal manometer and concluded that it underestimates work of breathing and hence should not be used as an ERT. ${ }^{23}$ Ferguson et al. found that PS SBT given according to ET size may overestimate extubation readiness. ${ }^{24}$ We used PS levels as per ET tube size like Randolph et al. and Gaffari et al., whereas Foronda et al. and Farias et al. used a uniform level of $10 \mathrm{~cm} \mathrm{H}_{2} \mathrm{O}$. ${ }^{12-14,19}$

Previous studies have shown that the majority of the ventilated patients do not require gradual weaning and can be extubated after an SBT, with 76 to $89 \%$ of patients passing it at first attempt. ${ }^{25-28}$ About $77.5 \%$ (31/40) passed the PS SBT in the first attempt in our study and $93.5 \%$ (29/31) of these were extubated. Faustino et al. in a study on 1,042 children ventilated for lower respiratory tract diseases found that $43 \%$ passed their first PS SBT of which $66 \%$ got extubated within the next 10 hours and $92.2 \%$ of them were successful. ${ }^{27}$ They found that PS SBT had a positive predictive value of $92 \%$ for successful extubation. The average duration at which PS SBT trial failed in our study was 23 minutes. Other studies have reported 20-45 minutes trial duration before failing. ${ }^{15,28}$

Our study found that protocol-guided daily screening, combined with a 2-hour PS SBT, did not significantly shorten the duration of $\mathrm{MV}$ or weaning time compared to conventional weaning.

Some adults studies have shown that identification of difficult to wean patients by use of a 2-hour T-piece SBT and then gradual 
Table 1: Characteristics of the study population

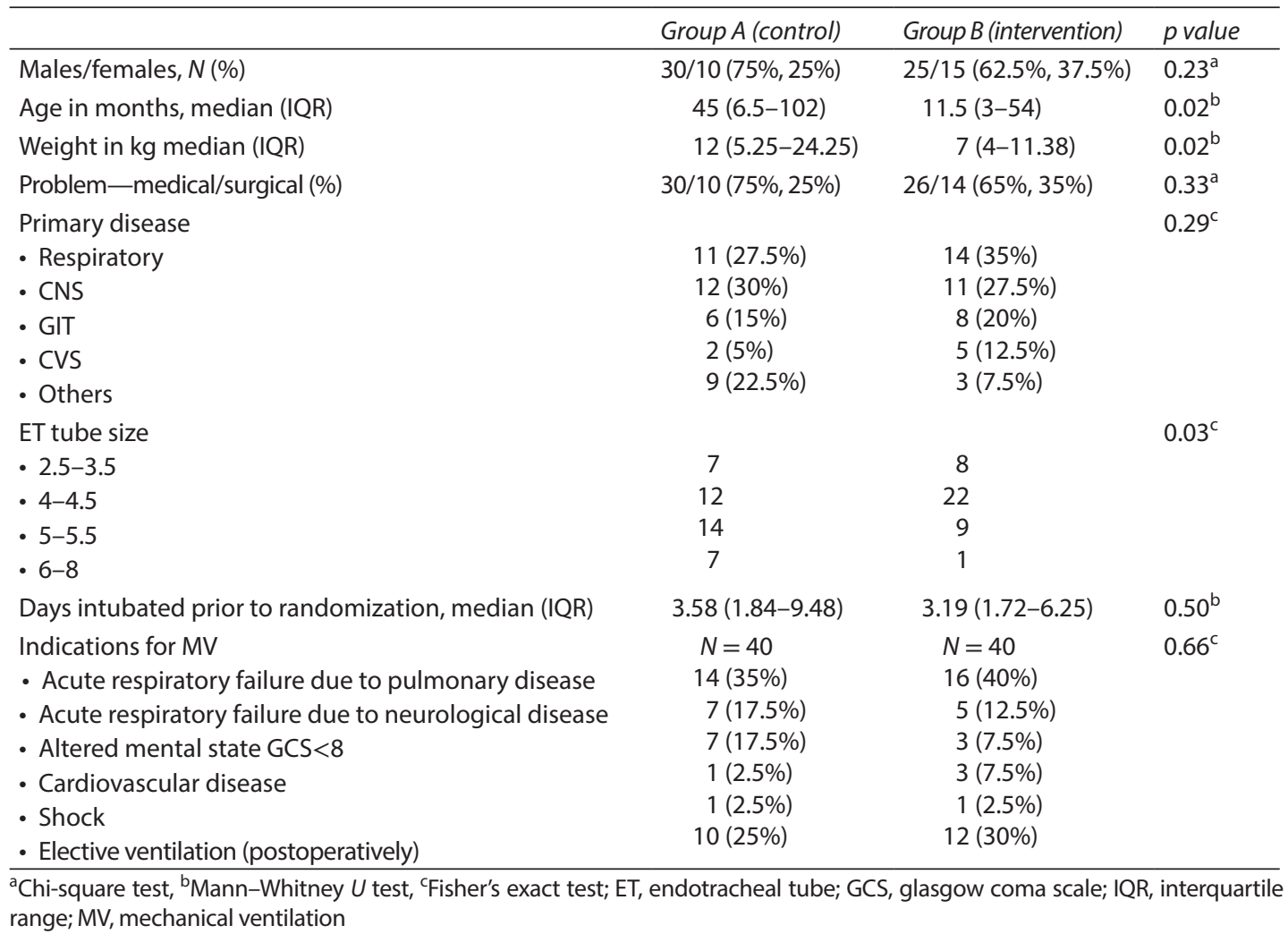

Table 2: Clinical profile of patients failing breathing trials

\begin{tabular}{|c|c|c|c|c|c|}
\hline Sl. No. & Diagnosis & $\begin{array}{l}\text { Failed } \\
\text { attempts }\end{array}$ & $\begin{array}{l}\text { Duration after } \\
\text { which trial failed }\end{array}$ & Cause for failure & $\begin{array}{l}\text { Days ventilated } \\
\text { prior to trial }\end{array}$ \\
\hline \multicolumn{6}{|c|}{ PS SBT failure in the intervention group $(N=9)$} \\
\hline 1 & Sepsis/pneumonia/pneumothorax & 1 & $15 \mathrm{~min}$ & Tachypnea & 2.58 \\
\hline 2 & $\begin{array}{l}\text { Corrosive poisoning/ } \\
\text { postgastrectomy }\end{array}$ & 1 & $15 \mathrm{~min}$ & Tachypnea & 3.00 \\
\hline 3 & $\begin{array}{l}\text { Sepsis/pneumonia/perforation peritonitis } \\
\text { (operated) }\end{array}$ & 1 & $1 \mathrm{hr}$ & $\begin{array}{l}\text { Low TV and } \\
\text { Tachypnea }\end{array}$ & 4.75 \\
\hline 4 & $\mathrm{ACHD} / \mathrm{VSD} / \mathrm{CHF}$ & 1 & $1 \mathrm{hr}$ & Tachypnea & 5.83 \\
\hline 5 & Coarctation of aorta (operated) & 1 & $15 \mathrm{~min}$ & Tachypnea & 8.00 \\
\hline 6 & Empyema & 2 & $10 \mathrm{~min}, 30 \mathrm{~min}$ & Tachypnea & 4.29 \\
\hline 7 & Posterior fossa mass/operated case & 4 & $\begin{array}{l}15 \mathrm{~min}, 10 \mathrm{~min} \\
30 \mathrm{~min}, 15 \mathrm{~min}\end{array}$ & $\begin{array}{l}\text { Low RR with apnea (on two } \\
\text { attempts) } \\
\text { Tachypnea and low TV (on two } \\
\text { attempts) }\end{array}$ & 1.75 \\
\hline 8 & $\begin{array}{l}\text { Post-traumatic meningitis with intracranial } \\
\text { infarcts }\end{array}$ & 4 & $10 \mathrm{~min}$ & Low RR & 2.50 \\
\hline 9 & Sepsis/empyema/shock & 4 & $15 \mathrm{~min}$ & Tachypnea & 5.67 \\
\hline \multicolumn{6}{|c|}{ T-piece trial failure in the control group $(N=3)$} \\
\hline 1 & $\mathrm{ACHD/VSD/PDA} / \mathrm{CHF}$ & 1 & $90 \mathrm{~min}$ & $\begin{array}{l}\text { Severe chest retractions } \\
\text { and } \mathrm{CO}_{2} \text { build-up }\end{array}$ & 1.08 \\
\hline 2 & Seizure disorder/aspiration pneumonia & 1 & $120 \mathrm{~min}$ & Seizure on T-piece & 1.29 \\
\hline 3 & Esophageal atresia (operated) & 1 & $120 \mathrm{~min}$ & Increased chest retractions & 3.00 \\
\hline
\end{tabular}

$\mathrm{ACHD}$, acyanotic congenital heart disease; $\mathrm{CHF}$, congestive heart failure; $\mathrm{PDA}$, patent ductus arteriosus; $\mathrm{RR}$, respiratory rate; $\mathrm{TV}$, tidal volume; $\mathrm{CO}_{2}$, carbon dioxide; VSD, ventricular septal defect 
Table 3: Comparison of outcome of two groups

\begin{tabular}{lll}
\hline & Group A (control) & Group B (intervention) \\
\hline Duration of mechanical ventilation in days & $N=38^{\mathrm{b}}$ \\
- Mean \pm SD & $N=38^{\mathrm{b}}$ & $5.72 \pm 4.6$ \\
- Median (interquartile range) & $6.78 \pm 5.7$ & $4.94(2.23-6.35)$ \\
Weaning time in hours & $4.77(2.89,9.46)$ & $N=38^{\mathrm{b}}$ \\
- Mean \pm SD & $N=38^{\mathrm{b}}$ & $32.68 \pm 39.27$ \\
- Median (interquartile range) & $36.98 \pm 35.02$ & $27(4.25-50.75)$ \\
Weaning time as percentage of total MV duration & $29.5(10.62-50)$ & $23.27 \%$ \\
Duration of PICU stay in days & $25.4 \%$ & $N=30^{\mathrm{c}}$ \\
- Mean \pm S.D & $N=32^{\mathrm{c}}$ & $19.77 \pm 18.78$ \\
- Median (interquartile range) & $16.87 \pm 11.91$ & $19.5(8.25,23)$ \\
Duration of hospital stay in days & $14(7.75,23.25)$ & $N=30^{\mathrm{d}}$ \\
- Mean \pm S.D & $N=31^{\mathrm{d}}$ & $26.83 \pm 19.75$ \\
- Median (interquartile range) & $27.77 \pm 23.56$ & $23.5(16.5,27.75)$ \\
\hline
\end{tabular}

${ }^{a}$ Mann-Whitney $U$ test; ${ }^{b}$ extubated patients; ${ }^{c}$ patients transferred out of PICU, ${ }^{d}$ discharged patients

protocolized weaning in them using different methods-SIMV, PSV, T piece, or daily T-piece SBT-did lead to a reduction in the duration of MV..$^{9,11,27,29}$ In children, two large studies have been conducted that showed opposite results. ${ }^{12,13}$ Randolph et al. used a PS SBT as a screening tool to either extubate children who passed the trial or randomize the one failing it to one of the three groups for protocolized weaning-PSV, volume support ventilation (VSV), or no protocol. ${ }^{12}$ They found that protocolized weaning did not shorten the ventilation duration. In contrast, Foronda et al. conducted daily screening to assess eligibility to give PS SBT, which was given only to the intervention group, like our study. ${ }^{13}$ They found that there was a reduction in the duration of $M V$ by a median of 1 day in children receiving protocolized weaning compared to conventional weaning.

On average, the weaning phase constitutes $40 \%$ of the total MV duration. ${ }^{6,27,28}$ In our study, it was $25.4 \%$ in SBT and $23.3 \%$ in control group ( $p=0.33$ ). This shorter weaning time could be because weaning was decided by a pediatric intensivist in our study instead of a respiratory therapist. We also did not find any difference in the weaning time in two groups like Randolph et al. ${ }^{12}$ However, Restrepo et al., Schulze et al., and a Cochrane review by Blackwood et al. found reduction in weaning time by protocolized weaning. ${ }^{30-33}$

The extubation failure rates were also similar between the intervention and control groups, i.e., $13 \%$ and $10.5 \%$, respectively. Randolph et al. and Foronda et al. found no difference in the extubation failure rate between the protocol and no protocol groups. ${ }^{12,13}$ The extubation failure rate reported in pediatric patients is quite heterogeneous and lies anywhere between $2.7 \%$ and $22 \%$ in various pediatric studies. ${ }^{1,3,19}$ Important to note is that the reintubated group had significantly higher mortality compared to the successfully extubated group, which had also been seen in previous adult and pediatric studies. ${ }^{15,19}$ According to a study by Kurachek et al., extubation failure leads to a fivefold increased risk of death in pediatric patients. ${ }^{1}$ He reported that nearly $83 \%$ of reintubation occurred within the first 12 hours. Average time for reintubation in our study was 5.6 hours in the control group and 17.5 hours in the intervention group.

We also had two patients who failed multiple PS SBTs but were still successfully extubated. Chavez et al. also found that while using CPAP of $5 \mathrm{~cm} \mathrm{H_{2 }}$ O as SBT, $9 \%$ of patients failed the trial of which half were still successfully extubated. ${ }^{33}$ Ferguson et al. reported that $2.7 \%(15 / 538)$ patients who underwent a PS SBT got extubated without passing it with only three extubation failures in them (20\%). ${ }^{24}$ Thus SBT is a reliable bedside test for assessing extubation readiness, but SBT failure does not always predict extubation failure.

The strengths of the study are that it was a prospective RCT and that the same pediatric intensivist assessed weaning and extubation readiness in all patients. Also, all patients were ventilated on the same mode of ventilation, i.e., pressure control SIMV. This study also had few limitations. This was a singlecenter study and the number of patients was small. The pediatric intensivist cannot be blinded in such studies to the type of weaning method being used. A larger sample size would give more conclusive results.

\section{ConClusion}

A significant number of patients could be extubated after passing PS SBT. Most of the patients who failed the SBT did so early (average being 23 minutes). However, the benefit of PS SBT in shortening the duration of ventilation was not seen in our study. Some patients failing the trial can still get successfully extubated with careful assessment and at the discretion of the intensivist. We did not find any serious events or adverse effects associated with PS SBT, e.g., accidental extubation, hemodynamic instability, or death. The extubation failure rate was similar in both groups. Mortality was seen to be higher in patients requiring reintubation compared to those successfully extubated.

\section{ACKnOWLedgments}

Dr Geetika Pant and Dr Josmy Jose, Senior Residents at PICU, Lok Nayak Hospital, for their assistance and cooperation during the study. Mr. Rajesh Ahuja for providing valuable statistical support.

Author Contributions: UJ was involved in the conceptualization of the study, project administration, formal analysis, supervision, critical review, and editing of the draft. RK was involved in the data acquisition, analysis, and drafting of the work.

\section{OrCID}

Rashmi Kishore 니 https://orcid.org/0000-0002-4668-1927 Urmila Jhamb ๑ https://orcid.org/0000-0003-1734-3504 


\section{References}

1. Kurachek SC, Newth CJ, Quasney MW, Rice T, Sachdeva RC, Patel NR, et al. Extubation failure in pediatric intensive care: a multiple-center study of risk factors and outcomes. Crit Care Med 2003;31(11):2657- 2664. DOI: 10.1097/01.CCM.0000094228.90557.85.

2. Newth CJ, Venkataraman S, Willson DF, Meert KL, Harrison R, Dean JM, et al. Weaning and extubation readiness in pediatric patients. Pediatr Crit Care Med 2009;10(1):1. DOI: 10.1097/PCC.0b013e318193724d.

3. Farias $J A$, Monteverde E. We need to predict extubation failure. $J$ Pediatr 2006;82(5):322-324. DOI: 10.2223/JPED.1539.

4. Esteban A, Frutos F, Tobin MJ, Alía I, Solsona JF, Valverdú I, et al. A comparison of four methods of weaning patients from mechanical ventilation. N Engl J Med 1995;332(6):345-350. DOI: 10.1056/ NEJM199502093320601.

5. Brochard L, Rauss A, Benito S, Conti G, Mancebo J, Rekik N, et al. Comparison of three methods of gradual withdrawal from ventilatory support during weaning from mechanical ventilation. Am J Respir Crit Care Med 1994;150(4):896-903. DOI: 10.1164/ajrccm.150.4.7921460.

6. MacIntyre NR, Cook DJ, Ely EW, Epstein SK, Fink JB, Heffner JE, et al. Evidence-based guidelines for weaning and discontinuing ventilatory support: a collective task force facilitated by the American College of Chest Physicians; the American Association for Respiratory Care; and the American College of Critical Care Medicine. CHEST 2001;120(Suppl. 6):375S-396S. DOI: 10.1378/chest.120.6_suppl.375s.

7. Valenzuela J, Araneda P, Cruces P. Weaning from mechanical ventilation in paediatrics. State of the art. Arch Bronconeumol 2014;50(3):105-112. DOI: 10.1016/j.arbres.2013.02.003.

8. Hess D.Ventilatormodes used in weaning. Chest2001;120(6):474S-476S. DOI: 10.1378/chest.120.6_suppl.474s.

9. Marelich GP, Murin S, Battistella F, Inciardi J, Vierra T, Roby M. Protocol weaning of mechanical ventilation in medical and surgical patients by respiratory care practitioners and nurses: effect on weaning time and incidence of ventilator-associated pneumonia. CHEST 2000;118(2):459-467. DOI: 10.1378/chest.118.2.459.

10. Kollef MH, Shapiro SD, Silver P, St John RE, Prentice D, Sauer S, et al. A randomized, controlled trial of protocol-directed versus physiciandirected weaning from mechanical ventilation. Crit Care Med 1997;25(4):567-574. DOI: 10.1097/00003246-199704000-00004.

11. Ely EW, Baker AM, Dunagan DP, Burke HL, Smith AC, Kelly PT, et al. Effect on the duration of mechanical ventilation of identifying patients capable of breathing spontaneously. N Engl J Med 1996;335(25):1864-1869. DOI: 10.1056/NEJM199612193352502.

12. Randolph AG, Wypij D, Venkataraman ST, Hanson JH, Gedeit RG, Meert KL, et al. Effect of mechanical ventilator weaning protocols on respiratory outcomes in infants and children: a randomized controlled trial. JAMA 2002;288(20):2561-2568. DOI: 10.1001/jama.288.20.2561.

13. Foronda F, Troster EJ, Farias JA, Barbas CS, Ferraro AA, Faria LS, et al. Impact of daily evaluation and spontaneous breathing test on the duration of pediatric mechanical ventilation: a randomized controlled trial.Crit Care Med 2011;15:1-31. DOI: 10.1097/CCM.0b013e3182257520.

14. Farias J, Retta A, Alia I, Olazarri F, Esteban A, Golubicki A, et al. A comparison of two methods to perform a breathing trial before extubation in pediatric intensive care patients. Intensive Care Med 2001;27(10):1649-1654. DOI: 10.1007/s001340101035.

15. Esteban A, Alia I, Gordo F, Fernández R, Solsona JF, Vallverdú I, et al. Extubation outcome after spontaneous breathing trials with T-tube or pressure support ventilation. Am J Respir Crit Care Med 1997;156(2):459-465. DOI: 10.1164/ajrccm.156.2.9610109.

16. Cabello B, Thille AW, Roche-Campo F, Brochard L, Gómez FJ, Mancebo J. Physiological comparison of three spontaneous breathing trials in difficult-to-wean patients. Intensive Care Med 2010;36(7):1171-1179. DOI: 10.1007/s00134-010-1870-0.

17. Chittawatanarat K, Orrapin S, Orrapin S. Open-label randomized control trial between low pressure support and T-piece method for discontinuation from mechanical ventilator and extubation in general surgical ICUs. Crit Care 2015;19(1):P268. DOI: 10.1186/cc14348.

18. Gnanapandithan K, Agarwal R, Aggarwal AN, Gupta D. Weaning by gradual pressure support (PS) reduction without an initial spontaneous breathing trial (SBT) versus PS-supported SBT: a pilot study. Rev Port Pneumol 2011;17(6):244-252. DOI: 10.1016/j. rppneu.2011.06.015.

19. Gaffari S, Ghasempour M, Bilan N. Spontaneous breathing trial a reliable method for weaning in children. Int J Pediatr 2015;3(3.2):707-712. DOI: 10.22038/ijp.2015.4424.

20. Bock KR, Silver P, Rom M, Sagy M. Reduction in tracheal lumen due to endotracheal intubation and its calculated clinical significance. CHEST 2000;118(2):468-472. DOI: 10.1378/chest.118.2.468.

21. Jarreau PH, Louis B, Dassieu G, Desfrere L, Blanchard PW, Moriette $\mathrm{G}$, et al. Estimation of inspiratory pressure drop in neonatal and pediatric endotracheal tubes. J Appl Physiol 1999;87(1):36-46. DOI: 10.1152/jappl.1999.87.1.36.

22. Takeuchi M, Imanaka H, Miyano H, Kumon K, Nishimura M. Effect of patient-triggered ventilation on respiratory workload in infants after cardiac surgery. Anesthesiology 2000;93(5):1238-1244. DOI: 10.1097/00000542-200011000-00017.

23. Khemani RG, Hotz J, Morzov R, Flink RC, Kamerkar A, LaFortune M, et al. Pediatric extubation readiness tests should not use pressure support. Intensive Care Med 2016;42(8):1214-1222. DOI: 10.1007/ s00134-016-4387-3.

24. Ferguson LP, Walsh BK, Munhall D, Arnold JH. A spontaneous breathing trial with pressure support overestimates readiness for extubation in children. Crit Care Med 2011;12(6):e330-e335. DOI: 10.1097/PCC.0b013e3182231220.

25. Farias JA, Alia I, Esteban A, Golubicki AN, Olazarri FA. Weaning from mechanical ventilation in pediatric intensive care patients. Intensive Care Med 1998;24(10):1070-1075. DOI: 10.1007/s001340050718.

26. Farias JA, Frutos F, Esteban A, Flores JC, Retta A, Baltodano A, et al. What is the daily practice of mechanical ventilation in pediatric intensive care units? A multicenter study. Intensive Care Med 2004;30(5):918-925. DOI: 10.1007/s00134-004-2225-5.

27. Faustino EVS, Gedeit R, Schwarz AJ, Asaro LA, Wypij D, Curley MA. Accuracy of an extubation readiness test in predicting successful extubation in children with acute respiratory failure from lower respiratory tract disease. Crit Care Med 2017;45(1):94-102. DOI: 10.1097/CCM.0000000000002024.

28. Boles JM, Bion J, Connors A, Herridge M, Marsh B, Melot C, et al. Weaning from mechanical ventilation. Eur Respir J 2007;29(5):1033-1056. DOI: 10.1183/09031936.00010206.

29. Esteban A, Anzueto A, Frutos F, Alía I, Brochard L, Stewart TE, et al. Characteristics and outcomes in adult patients receiving mechanical ventilation: a 28-day international study. JAMA 2002;287(3):345-355. DOI: 10.1001/jama.287.3.345.

30. Schultz TR, Lin RJ, Watzman HM, Durning SM, Hales R, Woodson A, et al. Weaning children from mechanical ventilation: a prospective randomized trial of protocol-directed versus physician-directed weaning. Respir Care 2001;46(8):772-782.

31. Restrepo RD, Fortenberry JD, Spainhour C, Stockwell J, Goodfellow L. Protocol-driven ventilator management in children: comparison to nonprotocol care. Intensive Care Med 2004;19(5):274-284. DOI: $10.1177 / 0885066604267646$.

32. Blackwood B, Murray M, Chisakuta A, Cardwell CR, O'Halloran P. Protocolized versus non-protocolized weaning for reducing the duration of invasive mechanical ventilation in critically ill paediatric patients. Cochrane Database Syst Rev 2013;7. DOI: 10.1002/14651858. CD009082.pub2.

33. Chavez A, dela Cruz R, Zaritsky A. Spontaneous breathing trial predicts successful extubation in infants and children. Pediatr Crit Care Med 2006;7(4):405. DOI: 10.1097/01.PCC.0000225001.92994.29. 